\title{
COMMENTARY
}

\section{The assessment and risk stratification of psychological morbidity in critical care survivors}

\author{
Joshua LC Wong and Stephen J Brett ${ }^{*}$ \\ See related research by Schandl et al., http://ccforum.com/content/17/5/R210
}

\begin{abstract}
Along with the ageing population, there is an expanding number of critical care survivors in the community. This group is at risk for both physical and psychological morbidity following their stay. Factors that predispose patients to commonly reported sequelae such as post-traumatic stress disorder, anxiety and depression remain incompletely elucidated. A tool to identify and stratify survivors at discharge may improve outcomes by allowing early and targeted intervention. Looking forward, the identification of aspects of practice associated with long-term adverse consequences should allow us to evolve our current protocols in a way that provides long-term benefit for patients.
\end{abstract}

With the recent focus on improving long-term quality of life for survivors of critical illness has come the requirement for practical ways of identifying people at risk of doing poorly [1]. Over the last decade or so it has been established beyond reasonable doubt that a significant proportion of patients who have survived admission to an ICU experience long-term psychological consequences. Such consequences can include anxiety, depression and post-traumatic stress disorder [2]. In addition many patients suffer from deficits in cognitive function that may be transient or prolonged [3].

What is less clear is the true proportion of individuals who experience these post-ICU phenomena. The literature that has formed our understanding of this proportion is predominantly from studies based around intensive care medicine follow-up clinics. Such studies are vulnerable to enrolment bias because the proportion

\footnotetext{
* Correspondence: Stephen.brett@imperial.ac.uk

Centre for Perioperative Medicine and Critical Care Research, Imperial College Health Care NHS Trust, Imperial College London, Hammersmith Hospital, Du Cane Road, London W12 OHS, UK
}

of patients attending clinics is often very modest. Follow-up studies thus often report only 10 to $20 \%$ of the potentially available population and there must therefore be substantial imprecision around estimates of the prevalence of post-intensive care discharge phenomena. Of course at an individual level we cannot anticipate why one patient may turn up for their clinic appointment and another not. From our own experience it seems that many patients do not return to clinic because they are either too well and have returned to work, or they feel too unwell and prefer not to return to hospital. This may be exacerbated by those individuals who are experiencing post-traumatic stress phenomena in whom avoidance is a major feature.

An important exception to this general summary of the literature is the paper by Dorothy Wade and colleagues published last year in Critical Care [4]. This paper was exceptional both in its thoroughness and in its retention of patients in their initial enrolled cohort for the duration of the study. We can thus have some confidence in the precision of their observations around the identification of risk factors. In many respects their study design is a useful model for future work, but it is resource intensive and hinges around establishment of firm relationships between researchers (or clinicians) and patients and their families whilst they are still very much part of the intensive care population.

In the United Kingdom, the National Institute for Health \& Clinical Excellence established a guideline for the rehabilitation of critically ill patients some time ago $[5,6]$. One of the major issues identified in this extensive review (which was chaired by one of the authors of this commentary, SJB) was that we lacked practicable tools to identify patients during their hospital stay who were at risk of poor quality rehabilitation and recovery.

The paper by Schandl and colleagues, to which this current commentary is linked [1], describes the early stages of development of a novel tool with which critical care survivors might be stratified at discharge into 
groups at varying risk of subsequent psychological morbidity. Risk groups were thus identified by regression analysis in a prospectively collected cohort of mixed intensive care patients. Data were assimilated during admission and on departure from critical care, and psychological morbidity (anxiety, depression and posttraumatic stress disorder) was assessed by a structured questionnaire 2 months after ICU discharge. Twentyone candidate variables were identified based on previous literature and a conceptual model that is not thoroughly described in the paper. A multiple regression model was developed with six factors subsequently identified as being significantly associated with risk of poor outcome. Based on post-hoc classification of risk into low-risk, medium-risk and high-risk groups, psychological morbidity was experienced in $12 \%, 50 \%$ and $63 \%$ of these groups, respectively.

For a training dataset, the receiver operating characteristic curve in the paper seems reasonably impressive. However, what this paper lacks is a prospective application of the model to a novel validation dataset. As the authors point out in their conclusion, this is needed before we can have real confidence in their observations. In addition, the outcomes were determined using frequently deployed screening questionnaires, The Hospital Anxiety and Depression Scale and the Post Traumatic Stress Scale - 10, which arguably may be a little oversensitive in this population.

The utility of any predictive tool is underpinned by there being some modification of treatment that could be applied, which would beneficially alter outcomes. The predictive factors identified by Schandl and colleagues seem to hinge largely on elements determined prior to an ICU survivor's admission: major pre-existing disease, being a parent to children younger than 18 years of age, previous psychological problems, being unemployed or on sick leave at ICU admission, as well as agitation and appearing depressed in the ICU. Wade and colleagues came at the problem from a somewhat different direction [4]. Their study identified rather more factors that might be tractable during an ICU stay - but things were described and measured differently and there was probably overlap between the studies in terms of a priori potential risk factors and outcome measures; overall, many of the outcome measures themselves move in the same direction - that is, they are co-linear [7]. This colinearity illustrates the difficulties of drawing this literature together in a way that informs future design of studies or indeed clinical systems [7].

Whilst Schandl and colleagues have made some headway in assimilating their own data into a clinically applicable tool, the lack of validation and the identification of new factors leave it firmly in the development stage. However, the ability to identify those survivors at significant risk may benefit them by allowing for increased surveillance and early intervention to reduce subsequent morbidity. Furthermore, the identification of risk factors that are associated with particular treatments or protocols should allow us to modify what we do to reduce adverse long-term consequences.

\section{Competing interests}

The authors declare that they have no competing interests.

\section{Acknowledgments}

The research was supported by the National Institute for Health Research (NIHR) Biomedical Research Centre based at Imperial College Healthcare NHS Trust and Imperial College London. The views expressed are those of the authors and not necessarily those of the National Health Service, the NIHR or the Department of Health.

\section{Published: 03 Oct 2013}

\section{References}

1. Schandl A, Bottai M, Hellgren E, Sundin O, Sackey PV: Developing an early screening instrument for predicting psychological morbidity after critical illness. Crit Care 2013, 17:R210.

2. Sukantarat K, Greer S, Brett S, Williamson R: Physical and psychological sequelae of critical illness. Br J Health Psychol 2007, 12:65-74.

3. Mikkelsen ME, Christie JD, Lanken PN, Biester RC, Thompson BT, Bellamy SL, Localio AR, Demissie E, Hopkins RO, Angus DC: The adult respiratory distress syndrome cognitive outcomes study: long-term neuropsychological function in survivors of acute lung injury. Am J Respir Crit Care Med 2012, 185:1307-1315.

4. Wade DM, Howell DC, Weinman JA, Hardy RJ, Mythen MG, Brewin CR, BorjaBoluda S, Matejowsky CF, Raine RA: Investigating risk factors for psychological morbidity three months after intensive care: a prospective cohort study. Crit Care 2012, 16:R192.

5. Tan T, Brett SJ, Stokes T, Guideline Development Group: Rehabilitation after critical illness: summary of NICE guidance. BMJ 2009, 338:b822.

6. Rehabilitation after Critical Illness. [http://www.nice.org.uk/cg83].

7. Wade D, Hardy R, Howell D, Mythen M: Identifying clinical and acute psychological risk factors for PTSD after critical care: a systematic review. Minerva Anestesio/ 2013, 79:944-963.

\section{$10.1186 / \mathrm{cc} 13039$}

Cite this article as: Wong and Brett: The assessment and risk stratification of psychological morbidity in critical care survivors. Critical Care 2013, 17:199 